\title{
THE HANF NUMBER OF THE FIRST ORDER THEORY OF BANACH SPACES
}

BY

\author{
SAHARON SHELAH AND JACQUES STERN
}

\begin{abstract}
In this paper, we discuss the possibility of developing a nice i.e. first order theory for Banach spaces: the restrictions on the set of sentences for recent compactness arguments applied to Banach spaces as well as for other model-theoretic results are both natural and necessary; without them we essentially get a second order logic with quantification over countable sets. Especially, the Hanf number for sets of sentences of the first order theory of Banach spaces is exactly the Hanf number for the second order logic of binary relations (with the second order quantifiers ranging over countable sets).
\end{abstract}

In recent years, värious authors have tried to develop a first order theory of Banach spaces and have obtained several successful results [2], [3], [6]. Nevertheless, they could not find a complete analogy with first order logic. Either, they had to restrict themselves to a proper subset of the set of all formulas of the first order language they considered, ${ }^{1}$ or else their results applied to classes of normed spaces and did not yield specific information on the Banach spaces included in these classes.

It is intuitively clear that the notion of Banach space is not first-order as it involves quantification over sequences. The present paper is an attempt to measure the gap between this notion and what can be expressed by first order logic. Essentially, we show that if one adds to a first order language suitable to discuss normed spaces a single formula meaning

"Every Cauchy sequence has a limit", then one gets all the strength of second order logic where all second order variables range over countable sets. Especially, it becomes possible to interpret the notion of well ordering.

Let $L$ be a first order language with equality which includes, besides variables, a binary function symbol + and a unary predicate symbol $B$. Any

Received by the editors July 13, 1976 and, in revised form, June 1, 1977.

AMS (MOS) subject classifications (1970). Primary 02H10, 46B99.

${ }^{1}$ The kind of restrictions which are necessary can be understood even by the reader not familiar with the model theory of Banach spaces: suppose one wants to imitate the proof of the compactness theorem for first order logic by ultraproducts. Then one has to take an ultraproduct of Banach spaces. Obviously this is not a real Banach space, so one has to omit the elements with "infinite" norm and to divide by the set of elements with infinitely small norm. Then, one gets a Banach space and for the sentences preserved under the above two operations one gets compactness. 
real Banach space $E$ can be viewed as an $L$-structure: + is interpreted by the addition in $E$ and $B$ by the unit ball of $E . L$ is clearly the weakest meaningful language to discuss Banach spaces.

1. MAIN THEOREM. There exists a set of closed formulas (sentences) of $L$, say $\Phi$ and formulas $\varphi(x), \psi(x, y), \theta$ such that

$\varphi(x)$ has one free variable;

$\psi(x, y)$ has two free variables;

$\theta$ is closed;

and

(1) Given a Banach space $E$ which is a model of $\Phi$, there is an infinite linear ordering $C(E)$ whose domain is the quotient of $\{a \in E: \varphi(a)\}$ by the equivalence relation $a= \pm b$, and whose order relation is defined by $\psi(a, b)$.

(2) Any infinite linear ordering is isomorphic to $C(E)$ for a suitable Banach space $E$ modelling $\Phi$.

(3) $C(E)$ is a well ordering if and only if $\theta$ holds in $E$.

The main theorem is a particular case of a more general result which applies not only to the notion of well ordering but to any property which can be expressed by a formula of a second order language, where second order variables are interpreted by countable sets. We will not state this result in its general form but we will restrict our attention to the set $L^{\prime}(\omega)$ of all second order formulas of the language $L^{\prime}$ which contains (besides equality) the only two-place predicate symbol $R$.

The notion of satisfaction is extended to formulas of $L^{\prime}(\omega)$ by interpreting second order variables by (at most) countable sets.

2. General Theorem. There exists a set of closed formulas of $L$, say $\Phi^{\prime}$, formulas $\varphi^{\prime}(x), \psi^{\prime}(x, y)$, and a mapping $\Theta: L^{\prime}(\omega) \rightarrow L$ such that:

$\varphi^{\prime}(x)$ has one free variable;

$\psi^{\prime}(x, y)$ has two free variables;

for any closed formula $\gamma$ of $L^{\prime}(\omega), \Theta(\gamma)$ is closed; and

(1) Given a Banach space $E$ which is a model of $\Phi^{\prime}$ there is an infinite $L^{\prime}$ structure $C(E)$ whose domain is the quotient of $\left\{a \in E: \varphi^{\prime}(a)\right\}$ by the equivalence relation $a= \pm b$ and whose binary relation is defined by $\psi^{\prime}(a, b)$.

(2) Any infinite $L^{\prime}$ structure with an infinite domain is of the form $C(E)$ for a suitable Banach space $E$ modelling $\Phi^{\prime}$.

(3) For any closed formula $\gamma$ in $L^{\prime}(\omega), C(E) \vDash \gamma$ if and only if $E \vDash \Theta(\gamma)$.

Furthermore any Banach space $E$ which is a model of $\Phi^{\prime}$ is the closed linear span of a set of linearly independent elements which has the same cardinality as $\left\{a \in E: \varphi^{\prime}(a)\right\}$, and is not the closed linear span of any set of smaller cardinality. 
That this result is essentially the best approximation to the notion of completeness is shown by the following.

3. THEOREM. There exists a closed formula $\gamma_{0}$ of $L(\omega)$ such that a normed space is a model of $\gamma_{0}$ if and only if it is a Banach space.

(By $L(\omega)$ we mean the set of formulas of the second order language associated with $L$; second order variables still range over countable sets.)

Before we describe in some detail the organization of the paper, let us discuss the consequences of the results on the computation of Hanf numbers.

Let $\mathcal{L}$ be an arbitrary language such that the class of sentences of $\mathcal{L}$ is actually a set. If $\psi$ is a sentence of $\mathcal{L}$, we let $K(\psi)$ be the class of all cardinals $\kappa$ such that $\psi$ has a model of cardinality $\kappa$. If $\Phi^{\prime}$ is a set of sentences, we define $K(\Phi)$ similarly. Now, for a given $\psi, K(\psi)$ may be bounded or not. The Hanf number of $\mathcal{L}$ is the first cardinal which exceeds all elements of $K(\psi)$ for any sentence $\psi$ of $\mathcal{L}$ such that $K(\psi)$ is bounded. In other words the Hanf number of $\mathcal{L}, h(\mathcal{L})$, is the least cardinal $\lambda$ such that, for any sentence $\psi$ of $\mathcal{L}$, the following holds: if $\psi$ has a model of power $\geqslant \lambda$, then $\psi$ has models of arbitrarily large cardinalities. Similarly, the Hanf number for sets of sentences of $\mathcal{L}, h^{\prime}(\mathcal{L})$, is the least cardinal $\lambda$ such that for any set of sentences $\Phi$ of $\mathcal{L}$, the following holds: if $\Phi^{\prime}$ has model of power $\lambda$, then $\Phi^{\prime}$ has models of arbitrarily large cardinalities.

Similarly, if $\psi$ (resp. $\Phi^{\prime}$ ) is a sentence (resp. a set of sentences) of $L$, we let $K_{B}(\kappa)$ (resp. $\left.K_{B}\left(\Phi^{\prime}\right)\right)$ be the class of all cardinals $\kappa$ such that $\psi\left(\right.$ resp. $\Phi^{\prime}$ ) has a model which is a Banach space with a dense subset of cardinality $\kappa$ and no dense subset of a smaller cardinality.

Hanf numbers are defined accordingly.

For example, the Hanf number of the first order theory of Banach spaces $h_{B}(L)$ is the least cardinal $\lambda$ such that, for any sentence of $L$ say $\psi, K(\psi)$ is unbounded if and only if $K(\psi)$ has an element $\geqslant \lambda$. The Hanf number for sets of sentences $h_{B}^{\prime}(L)$ is defined similarly.

Now, if $\Theta, \Phi^{\prime}$ are in the statement of the general theorem, then given a sentence $\psi$ of $L^{\prime}(\omega)$, one gets

$$
K(\psi)=K_{B}\left(\{\Theta(\psi)\} \cup \Phi^{\prime}\right) .
$$

Similarly if $\Psi$ is a set of sentences of $L^{\prime}(\omega)$

$$
K(\Psi)=K_{B}\left(\Theta(\Psi) \cup \Phi^{\prime}\right) .
$$

Also if $\psi$ is any sentence of $L$, by Theorem 3

$$
\forall \kappa \in K_{B}(\psi) \quad \exists \theta \in K\left(\psi \wedge \gamma_{0}\right) \quad \kappa \leqslant \theta \leqslant \kappa^{\kappa_{0}} .
$$

and if $\Psi$ is a set of sentences of $L$

$$
\forall \kappa \in K_{B}(\Psi) \quad \exists \theta \in K\left(\Psi \cup\left\{\gamma_{0}\right\}\right) \quad \kappa<\theta \leqslant \kappa^{\kappa_{0}} .
$$


From these observations, it is easy to derive the following inequalities:

(i) $h\left(L^{\prime}(\omega)\right)<h_{B}^{\prime}(L)$,

(ii) $h^{\prime}\left(L^{\prime}(\omega)\right) \leqslant h_{B}^{\prime}(L)$,

(iii) $h_{B}(L)<h\left(L^{\prime}(\omega)\right)$,

(iv) $h_{B}^{\prime}(L)<h^{\prime}\left(L^{\prime}(\omega)\right)$.

Thus:

4. THEOREM. The Hanf number for sets of sentences of the first order theory of Banach spaces is exactly the Hanf number for sets of sentences of the second order logic of binary relations (with the second order quantifiers ranging over countable sets).

Unfortunately, it is not possible to extract from Theorem 4 an exact "computation" of $h_{B}^{\prime}(L)$. As is well known [7], the Hanf numbers of languages which allow quantification over countable sets are "large cardinals" and their size are governed by large cardinals axioms. More precisely, Silver [8] has shown that the Hanf numbers of languages which express the notion of well ordering (such as $L^{\prime}(\omega)$ ) exceed the first cardinal $\kappa$ such that the partition property $\kappa \rightarrow(\omega)^{<\omega}$ holds, if such a cardinal exists. (Recall that $\kappa \rightarrow(\omega)^{<\omega}$ means that for any function $f$ defined on the set of finite subsets of $\kappa$ with value in $\{0,1\}$, there exists an infinite subset $X$ of $\kappa$, such that for each $n, f$ is constant on the set of (unordered) $n$-tuples from $X$.)

In the other direction, it is not difficult to see that both $h(L(\omega))$ and $h^{\prime}(L(\omega))$ are smaller than the Hanf number of the language $L_{\omega_{1} \omega_{1}}$ which allows countable conjunctions and disjunctions as well as countable strings of quantifiers. (For details see [7].) It is known, also (see [7]), that the Hanf number of $L_{\omega_{1} \omega_{1}}$ is smaller than the first strongly compact cardinal (if such a cardinal exists). This gives an upper bound for $h_{B}(L)$.

Going back to the main result, let us also point out that, although we do not mention them in the present paper, the real-valued languages defined by Krivine [2] and the second named author [3] have the same pathologies provided one admits alternation of quantifiers: theorems similar to those stated above can be proved for these languages and especially one can still interpret the notion of well ordering.

The paper is essentially devoted to the proof of the main theorem which goes as follows.

In $\S 1$, we construct a basic model $M$, which is a Banach space "encoding" the ordering of the set of rational numbers $Q$ as well as the infinite descending chains of this ordering. To perform the construction of $M$, we use a technique introduced by the second named author in order to solve the so-called problem of envelopes [5].

Next, in §2, we study the subspaces of the ultrapowers of $M$ and show that 
many properties of these subspaces can be expressed by formulas of $L$.

Finally, in \$3, we single out by a set of closed formulas $\Phi$ of $L$, specific subspaces of spaces $M^{I} / \mathcal{Q}$ and we prove the main theorem.

In $\S 4$, we briefly indicate how to adapt the preceding proof in order to get the general theorem and we prove Theorem 3.

The present paper grew out of conversations we had during the logic Colloquium held in Clermont-Ferrand in the summer of 1975. We are grateful to the Organizing Committee of this Colloquium for having invited both of us.

1. The basic model $M$. Let $\mathcal{I C}$ be an infinite dimensional real Hilbert space. The inner product is denoted by $(x, y)$ and the norm by $|x|=(x, x)^{1 / 2}$. If $e$ is an element of $\mathcal{H}$ with norm 1 , and $\delta$ a strictly positive real number we let $H(e, \delta)$ be the set of elements $x$ of $\mathcal{H}$ such that $(x, e)=1-\delta$; we let $F(e, \delta)$ be the set of elements $x$ of $H(e, \delta)$ such that $|x|<1$, a set $F(e, \delta)$ will be called a facet. Finally a segment $\left[x, x^{\prime}\right]$ is $\{y: \exists \theta \in[0,1] ; y=\theta x+(1$ $\left.-\theta) x^{\prime}\right\}$. (All these definitions are taken from [5].)

Now, let $A$ be a set of elements of norm one in $\mathcal{K}$, let $\delta$ be a strictly positive real and $\Delta$ be a function from $A$ into $\mathbf{R}$ such that

$$
\forall a \in A, \quad 0<\Delta(a)<\delta .
$$

Assume that the facets $F(\varepsilon a, \Delta(a)), \varepsilon= \pm 1, a \in A$, are pairwise disjoint, then, we define the space $\mathcal{H}(A, \Delta, \delta)$ to be $\mathcal{H}$ endowed with a new norm whose unit ball is the set of points $x$ such that

$|x|<1$, $|(x, a)|<1-\Delta(a), a \in A$.

It is easy to check that the unit sphere of $\operatorname{TC}(A, \Delta, \delta)$ is the union of the facets $F(\varepsilon a, \Delta(a)), \varepsilon= \pm 1, a \in A$, and of those elements $x$ with euclidian norm one such that $[0, x]$ does not meet any of the facets $F(\varepsilon a, \Delta(a))$.

$M$ is a space $\mathcal{H}(A, \Delta, \delta)$ for suitable $\mathcal{H}, A, \Delta, \delta$.

We first describe $\mathcal{H C} ; \mathcal{K}$ is the space $l^{2}(\mathbf{Q})$ where $\mathbf{Q}$ is the set of rational numbers. Recall that $l^{2}(X)$ is the set of mappings $u$ from $X$ to $\mathbf{R}$ such that $\Sigma_{x \in X}|u(x)|^{2}<\infty$, endowed with the inner product norm $\left(\Sigma_{x \in X}|u(x)|^{2}\right)^{1 / 2}$. We let $p, q, r, \ldots$ range over the rational numbers, $\sigma, \tau, \ldots$ over the set of finite strictly decreasing sequences of rational numbers denoted by $\mathbf{Q}^{<\omega} . e_{q}$ is the element of $\mathcal{H}$ whose value at $q$ is 1 and whose value elsewhere is 0 . If $\sigma=\left\{q_{1}, \ldots, q_{n}\right\}$ with $q_{1}>\cdots>q_{n}$, then we let

$$
a_{\sigma}=\left(e_{q_{1}}+\frac{e_{q_{2}}}{2}+\cdots+\frac{e_{q_{n}}}{n}\right)\left(\sum_{i=1}^{n} \frac{1}{i^{2}}\right)^{-1 / 2} .
$$

We let $l(\sigma)$ be the length of $\sigma$, i.e. $l(\sigma)=n$.

$A$ consists of the following points of $\mathcal{I C}$ : 


$$
\begin{aligned}
& e_{q}, q \in \mathbf{Q} . \\
& \left(e_{p}+2 \varepsilon e_{q}\right) / \sqrt{5}, p, q \in \mathbf{Q}, \varepsilon= \pm 1, p<q . \\
& a_{o}, \sigma \in \mathbf{Q}^{<\omega}, l(\sigma)>3 .
\end{aligned}
$$

Let $K$ be defined by $K=\left(\sum_{i=1}^{\infty} 1 / i^{2}\right)^{-1 / 2}=\sqrt{6} / \pi$.

LEMMA 1.1. Let $a, b$ be elements of $A$ distinct from all $a_{\sigma}, \sigma \in Q^{<\omega}$, $l(\sigma)>3$; let $\sigma, \tau$ be distinct elements of $\mathbf{Q}^{<\omega}$ of length $>3$. The following inequalities hold

(i) $|a \pm b|>1-2 / \sqrt{5}$;

(ii) $\left|a_{0} \pm a\right|>K / 3$;

(iii) $\left|a_{0} \pm a_{\tau}\right|>K / n(\sigma, \tau)$

where $\sigma=\left(q_{1}, \ldots, q_{n}\right), \tau=\left(q_{1}^{\prime}, \ldots, q_{m}^{\prime}\right)$ and $n(\sigma, \tau)$ is the least integer $i$ such that $q_{i} \neq q_{i}$, or $i>n$, or $i>m$.

Proof. We leave to the reader the computations needed to prove (i) and (ii), and we turn to (iii). Let $\sigma=\left\{q_{1}, \ldots, q_{n}\right\}, q_{1}>\cdots>q_{n}$ and $\tau=$ $\left\{q_{1}^{\prime}, \ldots, q_{m}^{\prime}\right\}, q_{1}^{\prime}>\cdots>q_{m}^{\prime}$. Assume $i$ is the least integer such that $q_{i} \neq q_{i}^{\prime}$ or $q_{i}$ (resp. $q_{i}^{\prime}$ ) is undefined. We may assume without loss of generality

either that $q_{i}>q_{i}^{\prime}$; or

that $q_{i}$ is defined and $q_{i}^{\prime}$ undefined.

In both cases, $q_{i} \in \sigma, q_{i} \notin \tau$.

We have

$$
\begin{aligned}
& \left(a_{0}, e_{q_{i}}\right)=\frac{1}{i}\left(\sum_{j=1}^{n} \frac{1}{j^{2}}\right)^{-1 / 2}>\frac{K}{i}, \\
& \left(a_{\tau}, e_{q_{1}}\right)=0
\end{aligned}
$$

therefore

$$
\left(a_{\sigma}-a_{\tau}, e_{q}\right)>K / i, \quad\left(a_{\sigma}+a_{\tau}, e_{q}\right)>K / i
$$

so that

and similarly

$$
\left|a_{0}-a_{r}\right|>K / i
$$

$$
\left|a_{\sigma}+a_{\tau}\right|>K / i \text {. Q.E.D. }
$$

LEMMA 1.2. Let $F(e, \gamma)$ be a facet in $\mathcal{Y}$; any point $x$ of $F(e, \gamma)$ satisfies $|x-e|<\sqrt{2 \gamma}$.

Proof. $|x-e|^{2}=|x|^{2}+|e|^{2}-2(x, e)<2-2(1-\gamma)=2 \gamma$.

In order to define the function $\Delta$, we assume $\sqrt{2 \delta}<K / 9$, $\sqrt{2 \delta}<\frac{1}{3}(1-2 / \sqrt{5})$ and $\delta \in Q$, and we choose an infinite strictly decreasing sequence $\left(\delta_{n}\right)_{n \in N}$ such that:

$$
\delta_{0}=\delta, \sqrt{2 \delta_{n}}<K / 3(n+1)
$$


$\left(\delta^{2} /(n+1)^{2}\right.$ is such a sequence).

We remark that $\lim _{n \rightarrow \infty} \delta_{n}=0$.

We now let

$$
\begin{aligned}
& \Delta\left(e_{q}\right)=\delta_{0} ; q \in \mathbf{Q} . \\
& \Delta\left(\left(e_{p}+2 \varepsilon e_{q}\right) / \sqrt{5}\right)=\delta_{1}, \varepsilon= \pm 1, p<q . \\
& \Delta\left(a_{\sigma}\right)=\delta_{n} ; \sigma \in \mathbf{Q}^{<\omega}, l(\sigma)=n>3 .
\end{aligned}
$$

We have to check that this is a correct definition.

LemMA 1.3. The facets $F(\varepsilon a, \Delta(a)), \varepsilon= \pm 1, a \in A$, are pairwise disjoint.

Proof. In view of Lemma 1.2, it is enough to show that given any two distinct elements of $A$, say $a$ and $a^{\prime}$ we have

$$
\left|a \pm a^{\prime}\right|>\sqrt{2 \Delta(a)}+\sqrt{2 \Delta\left(a^{\prime}\right)} .
$$

We now use the inequalities in Lemma 1.1. If $a$ and $a^{\prime}$ are distinct from all $a_{\sigma}$ 's, we get by inequality (i)

$$
\left|a \pm a^{\prime}\right|>1-2 / \sqrt{5}>3 \sqrt{2 \delta}>\sqrt{2 \Delta(a)}+\sqrt{2 \Delta\left(a^{\prime}\right)} \text {. }
$$

In case only one of $a, a^{\prime}$ is some $a_{\sigma}$, we apply inequality (ii). Finally if $a=a_{\sigma}$ and $a^{\prime}=a_{\tau}$, then $\left|a_{\sigma}-a_{\tau}\right|>K / n(\sigma, \tau)$, where $n(\sigma, \tau)$ is the first integer $i$ such that $q_{i} \neq q_{i}^{\prime}$ so that if $\sigma$ has length $n$ and $\tau$ has length $m, n(\sigma, \tau)<$ $\min (m, n)+1$, we get

$$
\left|a_{o}-a_{\tau}\right|>\frac{K}{3(n+1)}+\frac{K}{3(m+1)}>\sqrt{2 \delta_{n}}+\sqrt{2 \delta_{m}} .
$$

This finishes the proof of Lemma 1.3.

We now state and prove some properties of our basic space $M=$ $\operatorname{SC}(A, \Delta, \delta)$.

LEMMA 1.4. For any $x$ in $\mathcal{H}$, the following holds

$$
|x|<\|x\|<(1-\delta)^{-1}|x| \text {. }
$$

The equality $\|x\|=(1-\delta)^{-1}|x|$ holds if and only if $x=\lambda e_{q}$ for some $\lambda \in \mathbf{R}$ and some $q \in \mathbf{Q}$.

Proof. The inequality $|x|<\|x\|$ follows from the fact that the unit ball of $\operatorname{IC}(A, \Delta, \delta)$ is included in the unit ball of the euclidian norm. To check the inequality $\|x\|<(1-\delta)^{-1}|x|$ it is enough to see that any element $x$ of the unit sphere $S$ of $\operatorname{SC}(A, \Delta, \delta)$ has euclidian norm $>1-\delta$. This is clear for those elements of $S$ with euclidian norm one; as for the other ones, they belong to one of the facets $F(\varepsilon a, \Delta(a)), \varepsilon= \pm 1, a \in A$; but it is easy to prove that if $x \in F(\varepsilon a, \Delta(a))$, then $|x|>1-\Delta(a)$, and $|x|=1-\Delta(a)$ only if $x$ is a multiple of $a$, so that $|x|=1$ if and only if $|x|=\varepsilon(1-\delta) e_{q}$. This finishes the proof. 
Actually, for many elements $x$, one gets $|x|=\|x\|$. Especially, the following result will be useful.

LEMMA 1.5. Assume $x$ is such that $\|x\|=1$, and $(1-\delta)^{-1} \sqrt{2 \delta}<\| x-$ $e_{q} \|<2 \sqrt{2 \delta}$ then $|x|=1$.

Proor. By Lemma 1.4, we get

$$
\sqrt{2 \delta}<\left|x-e_{q}\right|<2 \sqrt{2 \delta} .
$$

Now, if $|x| \neq 1$, then $x$ belongs to a facet $F(\varepsilon a, \Delta(a)), \varepsilon= \pm 1, a \in$ A. From Lemma 1.2, it follows that $a \neq e_{q}$, but, by Lemma 1.1., we get $\left|e_{q}-\varepsilon a\right|>$ $K / 3>3 \sqrt{2 \delta}$ and by Lemma $1.2,|x-\varepsilon a|<\sqrt{2 \delta}$. Hence we get $\left|e_{q}-x\right|>$ $2 \sqrt{2 \delta}$; contradiction.

Lemma 1.5 admits a kind of converse.

LEMMA 1.6. There exist fixed real numbers $\alpha, \beta$ such that

(1) $1>\alpha>\beta>0$.

(2) Any element $x$ such that $|x|=1$ and $\alpha>\left(x, e_{q}\right)>\beta$ satisfies $\|x\|=1$.

Proof. Assume $|x|=1$ and $\|x\| \neq 1$. Then there exists a facet $F(\varepsilon a, \Delta(a)$ ), $a \in A, \varepsilon= \pm 1$ such that $(x, \varepsilon a)>1-\Delta(a)$. Hence

$$
\begin{gathered}
|x-\varepsilon a|^{2}=2-2 \varepsilon(x, a)<2 \Delta(a), \\
|x-\varepsilon a|<2 \sqrt{\Delta(a)}<2 \sqrt{\delta} .
\end{gathered}
$$

If $\alpha=1-\delta$, the hypothesis $\alpha>\left(x, e_{q}\right)$ implies

$$
\left|x-e_{q}\right|^{2}=2-2\left(x, e_{q}\right)>2(1-\alpha)=2 \delta,
$$

so that $\left|x-e_{q}\right| \geqslant \sqrt{2 \delta}$ which, in turn, implies $\varepsilon a \neq e_{q}$. Now, by Lemma 1 , we get

$$
\left|e_{q}-\varepsilon a\right| \geqslant K / 3>3 \sqrt{2 \delta} .
$$

So $\left|x-e_{q}\right| \geqslant\left|e_{q}-\varepsilon a\right|-|\varepsilon a-x|>2 \sqrt{2 \delta}$. If $\beta=1-4 \delta$, then, the inequality $\left(x, e_{q}\right)>\beta$ implies

$$
\left|x-e_{q}\right|^{2}=2-2\left(x, e_{q}\right)<2(1-\beta)=8 \delta
$$

which contradicts

$$
\left|x-e_{q}\right|>2 \sqrt{2 \delta} .
$$

2. Ultrapowers of $M$ and their subspaces. We now turn to ultrapowers of $M$ and their subspaces. Detailed information about ultrapowers can be found in [1] or [3] or else [4]. For the reader's convenience, we recall some definitions. In what follows, $Q$ is an ultrafilter on a set $I$. If $\left(\alpha_{i}\right)_{i \in I}$ is a bounded family of real numbers, the $\operatorname{limit} \lim _{2} \alpha_{i}$ is the unique real number $\alpha$ such that for any $\varepsilon>0\left\{i:\left|\alpha_{i}-\alpha\right|<\varepsilon\right\} \in \mathcal{U}$. Now if $E$ is a Banach space, we let $\Pi_{0}$ be 
space defined by:

$$
\Pi_{0}=\left\{\left(x_{i}\right)_{i \in I} \in E^{I}: \text { for some } \lambda>0\left\|x_{i}\right\|<\lambda\right\} .
$$

$\Pi_{0}$ is endowed with the seminorm

$$
\left\|\left(x_{i}\right)_{i \in I}\right\|=\lim _{Q}\left\|x_{i}\right\| \text {. }
$$

The set $N$ of elements of $\Pi_{0}$ with seminorm 0 is a subspace of $\Pi_{0}$ and the ultrapower $E^{I} / \mathcal{Q}$ is the quotient space $\Pi_{0} / N$, which is a Banach space (see $[3$, \$6]).

In view of Lemma 1.4, it folows from the definitions that $M^{I} / \mathcal{U}$ and $\mathcal{H C}^{I} / \mathcal{U}$ are based on the same space so it is convenient to have a description of $M^{I} / \mathcal{Q}$ in terms of $\mathcal{C}^{I} / \mathcal{Q}$. To give this description, we let $\mathbf{Q}^{*}=\mathbf{Q}^{I} / \mathcal{U}$ be the ultrapower of $\mathbf{Q}$ in the usual sense; $\Im C^{I} / \mathcal{Q}$ is a Hilbert space: if $x$ is $\left(x_{i}\right)_{i \in I}$ and $y$ is $\left(y_{i}\right)_{i \in I}$, the inner product is given by $\lim _{Q}\left(x_{i}, y_{i}\right)$. If $q^{*} \in Q^{*}$, $e_{q^{*}}$ denotes the element $\left(e_{q^{*}(i)}\right)_{i \in I^{*}}$. If $\sigma^{*}$ is a finite decreasing sequence $q_{1}^{*}>\cdots>q_{n}^{*}$, then

$$
a_{\sigma^{*}}=\left(e_{q_{i}^{*}}+\cdots+\frac{e_{q_{i}^{*}}}{n}\right)\left(\sum_{i=1}^{n} \frac{1}{i^{2}}\right)^{-1 / 2} .
$$

Proposition 2.1. Let $\mathcal{Q}$ be an ultrafilter on a set $I, M^{I} / \mathcal{Q}$ is $\mathcal{I C}^{I} / \mathcal{U}\left(A^{\prime}, \Delta^{\prime}, \delta\right)$ where $A^{\prime}$ consists of the elements

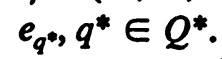

$\left(e_{p^{*}}+2 \varepsilon e_{q^{*}}\right) / \sqrt{5}, \varepsilon= \pm 1, p^{*}<q^{*}$.

$$
a_{0^{*}}, \sigma^{*} \in Q^{*<\omega}, l\left(\sigma^{*}\right)>3 \text {; }
$$

and $\Delta^{\prime}$ is defined by

$$
\begin{aligned}
& \Delta^{\prime}\left(e_{q^{*}}\right)=\delta_{0} ; \\
& \Delta^{\prime}\left(\left(e_{p^{*}}+2 \varepsilon e_{q^{*}}\right) / \sqrt{5}\right)=\delta_{1} ; \\
& \Delta^{\prime}\left(a_{\sigma^{*}}\right)=\delta_{n}, \text { if } \sigma^{*} \in Q^{*<\omega}, l\left(\sigma^{*}\right)=n>3 .
\end{aligned}
$$

Proof. We first prove that the unit ball of $M^{I} / \mathcal{U}$ is included in the unit ball of $\mathcal{T C}^{I}\left(A^{\prime}, \Delta^{\prime}, \delta\right)$. Once this is done we prove the reverse inclusion. Let $x$ be given by $\left(x_{i}\right)_{i \in I}$ and assume $\|x\|<1$ in the ultrapower of $M$, let $x_{i}^{\prime}=$ $x_{i} / \sup \left(1,\left\|x_{i}\right\|\right)$, then $\lim _{\text {ou }}\left\|x_{i}^{\prime}-x_{i}\right\|=0$, so that we may assume without loss of generality that $x$ is given by $\left(x_{i}\right)_{i \in I}$ with $\left\|x_{i}\right\|<1$. Now if $\left\|x_{i}\right\|<1$, then in $\mathcal{H}$ we have:

$$
\left|x_{i}\right|<1,\left|\left(x_{i}, a\right)\right|<1-\Delta(a), a \in A \text {. }
$$

Consider a given $q^{*}$ one gets

$$
\left|\left(x_{i}, e_{q^{*}(i)}\right)\right|<1-\Delta\left(e_{q^{*}(i)}\right)
$$

and therefore in $\mathcal{H}^{I} / \mathcal{U}$

$$
\left|\left(x, e_{q^{*}}\right)\right|<1-\Delta^{\prime}\left(e_{q^{*}}\right)
$$


Similarly

$$
\left|\left(x, \frac{e_{p^{*}}+2 \varepsilon e_{q^{*}}}{\sqrt{5}}\right)\right| \leqslant 1-\Delta^{\prime}\left(\frac{e_{p^{*}}+2 \varepsilon e_{q^{*}}}{\sqrt{5}}\right) .
$$

Now let $\sigma^{*}=\left(q_{1}^{*}, \ldots, q_{n}^{*}\right)$ be given with $q_{1}^{*}>\ldots>q_{n}^{*}$; then, $\left\{i\right.$ : $q_{1}^{*}(i)$ $\left.>\cdots>q_{n}^{*}(i)\right\} \in \mathcal{Q}$. Let $\sigma_{i}$ be $\left\{q_{1}^{*}(i), \ldots, q_{n}^{*}(i)\right\}$; then almost everywhere with respect to $\mathcal{Q}, \sigma_{i}$ is a decreasing sequence of length $n$ and $\left|\left(x_{i}, a_{\sigma_{i}}\right)\right|<1-$ $\delta_{n}=1-\Delta\left(a_{\sigma}\right)$. Clearly, $\left(a_{\sigma_{i}}\right)_{i \in I}$ is exactly $a_{\sigma^{*}}$ so that

$$
\left|\left(x, a_{\sigma^{*}}\right)\right|<1-\Delta^{\prime}\left(a_{\sigma^{*}}\right) \text {. }
$$

So far, we have proved that the unit ball of $M^{I} / \mathcal{Q}$ is included in the unit ball of $\mathcal{H}^{I} / \mathcal{U}\left(A^{\prime}, \Delta^{\prime}, \delta\right)$. We now prove the reverse inclusion. If this inclusion does not hold, we may pick $x=\left(x_{i}\right)_{i \in I}$ such that

$x$ has norm $<1$ in $\mathcal{H}^{I} / \mathscr{U}\left(A^{\prime}, \Delta^{\prime}, \delta\right)$,

$x$ has norm $>1$ in $M^{I} / \mathscr{Q}$.

Let $\|x\|=1+\eta$ in $M^{I} / Q, \eta>0$; on one hand $|x|$ is at most one so that one may assume that for all $i\left|x_{i}\right|<1$. On the other hand $\left\{i:\left\|x_{i}\right\|>1+\right.$ $\eta / 2\} \in \mathcal{Q}$; so that equivalently

$$
\left\{i:\left\|x_{i}(1+\eta / 2)^{-1}\right\|>1\right\} \in \mathcal{Q} \text {. }
$$

It follows that almost everywhere with respect to $\mathscr{U}$ there exists $a_{i} \in A$ such that

$$
\left|\left(x_{i}(1+\eta / 2)^{-1}, a_{i}\right)\right| \geqslant 1-\Delta\left(a_{i}\right)
$$

or

$$
\left|\left(x_{i}, a_{i}\right)\right|>(1+\eta / 2)\left(1-\Delta\left(a_{i}\right)\right)
$$

if $a$ is given by $\left(a_{i}\right)_{i \in I}$ (where $a_{i}$ is 0 when undefined), one gets

$$
|(x, a)| \geqslant\left(1+\frac{\eta}{2}\right)\left(1-\lim _{\mathcal{Q}} \Delta\left(a_{i}\right)\right) \text {. }
$$

We now distinguish four cases:

Case 1. $\left\{i: \Delta\left(a_{i}\right)=\delta_{0}\right\} \in \mathcal{Q}$. Then $a_{i}$ is almost everywhere equal to some $e_{q(i)}$, so that $a$ is equal to some $e_{q^{*}}$ and $\lim _{\mathcal{Q}} \Delta\left(a_{i}\right)=\Delta^{\prime}\left(e_{q^{*}}\right)$, but then inequality $(+)$ gives

$$
\left|\left(x, e_{q^{*}}\right)\right|>1-\Delta^{\prime}\left(e_{q^{*}}\right)
$$

and this contradicts the fact that $x$ has norm $<1$ in $\mathcal{H}\left(A^{\prime}, \Delta^{\prime}, \delta\right)$.

Case 2. $\left\{i: \Delta\left(a_{i}\right)=\delta_{1}\right\} \in Q$. Then, similarly, $a$ is equal to some $\left(e_{p^{*}}+\right.$ $\left.2 \varepsilon e_{q^{*}}\right) / \sqrt{5}$ and $\lim _{\mathcal{Q}} \Delta\left(a_{i}\right)=\Delta^{\prime}\left(\left(e_{p^{*}}+2 \varepsilon e_{q^{*}}\right) / \sqrt{5}\right)$; contradiction.

Case 3. For some $n \geqslant 3,\left\{i: \Delta\left(a_{i}\right)=\delta_{n}\right\} \in \mathcal{Q}$; then, almost everywhere with respect to $थ, a_{i}$ is equal to some $a_{\sigma(i)}$ with $l(\sigma(i))=n$; let 


$$
\sigma(i)=\left\{q_{1}(i)>\cdots>q_{n}(i)\right\}
$$

then, if $q_{j}^{*}$ is $\left(q_{j}(i)\right)_{i \in I}, 1<j \leqslant n$, and if $\sigma^{*}=\left\{q_{1}^{*}>\cdots>q_{n}^{*}\right\}$, we get

$$
a=a_{\sigma^{*}} \text { and } \lim _{\mathscr{U}} \Delta\left(a_{i}\right)=\Delta^{\prime}\left(a_{\sigma^{*}}\right) \text {. }
$$

Then, it follows from inequality $(+)$ that

$$
\left|\left(x, a_{\sigma^{*}}\right)\right|>1-\Delta^{\prime}\left(a_{\sigma^{*}}\right)
$$

this contradicts the assumption.

Case 4. Cases 1, 2, 3 fail. Then $\lim _{\mathrm{Q}} \Delta\left(a_{i}\right)=0$ so that $(+)$ becomes $|(x, a)|>1+\eta / 2$; this, in turn, gives $|x|>1$; contradiction.

This finishes the proof of Proposition 2.1.

Actually, we have not checked that the definition of $\mathcal{T C}^{I} / \mathcal{U}\left(A^{\prime}, \Delta^{\prime}, \delta^{\prime}\right)$ is correct. This can be done directly by proving the analogs of Lemmas 1.1, 1.2, 1.3; for example, the analog of Lemma 1.1 reads:

LEMMA 2.2. Let $a, b$ be distinct elements of $A^{\prime}$ distinct from all $a_{\sigma^{*}}$; let $\sigma^{*}, \tau^{*}$ be distinct elements of $\mathbf{Q}^{*<\omega}$; the following inequalities hold:

(i) $|a \pm b|>1-2 / \sqrt{5}$;

(ii) $\left|a_{\sigma^{*}} \pm a\right|>K / 3$;

(iii) $\left|a_{\sigma^{*}} \pm a_{\tau^{*}}\right| \geqslant K / n\left(\sigma^{*}, \tau^{*}\right)$;

where $\sigma^{*}=\left(q_{1}^{*}, \ldots, q_{n}^{*}\right), \tau^{*}=\left(q_{1}^{*}, \ldots, q_{m}^{\prime *}\right)$ and $n\left(\sigma^{*}, \tau^{*}\right)$ is the least integer $i$ such that $q_{i}^{*} \neq q_{i}^{* \prime}$, or $i>n$ or $i>m$.

Similarly, one can state and prove the analogs of Lemmas 1.4 and 1.6.

LEMMA 2.3. For any $x$ in $\mathcal{H}^{I} / \mathcal{Q}$, the following holds

$$
|x| \leqslant\|x\| \leqslant(1-\delta)^{-1}|x| \text {. }
$$

The equality $\|x\|=(1-\delta)^{-1}|x|$ holds if and only if $x=\lambda e_{q^{*}}$ for some $\lambda \in \mathbf{R}$ and some $q^{*} \in \mathbf{Q}^{*}$.

LEMMA 2.4. There exist fixed real numbers, $\alpha, \beta$ such that:

(1) $1>\alpha>\beta>0$

(2) Any element $x$ such that $|x|=1$ and $\alpha>\left(x, e_{q^{*}}\right)>\beta$ satisfies $\|x\|=1$.

We now restrict our attention to the class of all closed infinite dimensional subspaces $X$ of ultrapowers $M^{I} / थ$. Such a subspace $X$ is endowed with two norms: its own norm inherited from $M^{I} / \mathcal{Q}$ and the euclidian norm inherited from $\mathcal{H}^{I} / \mathcal{Q}$. We consider these $X$ as $L$-structures (the norm used being the one inherited from $M^{I} / Q$ ) and we show that a lot of information on $X$ can be expressed by formulas of $L$. To be more precise, assume one can associate to any $X$ an $n$-ary relation $R(X)$ over $X$ i.e. a subset of $X^{n}$; we say that $R$ is definable if there is a formula $\varphi\left(x_{1}, \ldots, x_{n}\right)$ of $L$ such that given any $X$ together with $n$-elements $b_{1}, \ldots, b_{n}$ of $X$ 


$$
X \vDash \varphi\left(b_{1}, \ldots, b_{n}\right) \text { if and only if }\left(b_{1}, \ldots, b_{n}\right) \in R(X) .
$$

We say $\varphi$ defines $R$.

We now study definable relations.

LEMMA 2.5. Let $q$ be a rational number; the relation $a=q b$ is definable.

Proof. Assume $q>0, q=m n^{-1}, m \in \mathrm{N}, n \in \mathrm{N}$; then $c=q b$ iff $n c=m b$ where $n c$ is defined by

$$
\frac{c+\cdots+c}{n \text { times }}
$$

If $q=0, c=q b$ is $c+c=c$. If $q<0, c=q b$ is equivalent to

$$
\exists x \quad(x+b=0 \text { and } c=(-q) x) .
$$

Corollary 2.6. Let $q, q_{1}, \ldots, q_{n}$ be elements of $\mathbf{Q}$. The relation

is definable.

$$
\left\|q_{1} b_{1}+\cdots+q_{n} b_{n}\right\|<q
$$

We leave the proof to the reader.

LEMMA 2.7. The set of elements $b$ of $X$ such that $\|b\|=|b|=1$ is the set of extreme points of the unit ball of $X$. Therefore, the relation $\|b\|=|b|=1$ is definable.

Proof. The set of extreme points of the unit ball is definable by the formula $\varepsilon(x)$

$$
B(x) \wedge \forall y \forall y^{\prime}\left(B(y) \wedge B\left(y^{\prime}\right) \wedge y \neq y^{\prime} \rightarrow y+y^{\prime} \neq 2 x\right) .
$$

Now, we claim that this set of extreme points is exactly $\{b:\|b\|=|b|=1\}$.

Clearly if $b$ is an extreme point $\|b\|=1$. Now, if we have $|b|<1$, then $b$ is an interior point of a facet $F\left(\varepsilon a, \Delta^{\prime}(a)\right), \varepsilon= \pm 1, a \in A^{\prime}$. As $X$ is infinite dimensional there exists a segment $\left[c, c^{\prime}\right]$ such that

$c \neq c^{\prime}$,

$\left[c, c^{\prime}\right] \subseteq X \cap F\left(\varepsilon a, \Delta^{\prime}(a)\right)$,

$b$ is an interior point of $\left[c, c^{\prime}\right]$.

This contradicts the fact that $b$ is extremal.

Conversely, if $\|b\|=|b|=1$, then $b$ is an extreme point; this is because $\|c\|<1$, and $\left\|c^{\prime}\right\|<1$, and $c \neq c^{\prime}$ imply $|c|<1$ and $\left|c^{\prime}\right|<1$ so that $\mid(c+$ $\left.c^{\prime}\right) / 2 \mid<1$ hence $\left(c+c^{\prime}\right) / 2 \neq b$.

We now try to express the relation $|b|=1$; we need a lemma:

LEMMA 2.8. Let $x, y$ be distinct elements of $X, 0<\theta<2 \pi / 5$; assume at least five distinct points $(\cos k \theta) x+(\sin k \theta) y, k \in \mathbf{N}$, are extreme points of the unit ball of $X$, then $|x|=|y|=1$. 
Proof. Let $P$ be the plane spanned by $x$ and $y$. The set of elements $z=(\cos \lambda) x+(\sin \lambda) y, \lambda \in[0,2 \pi]$, is an ellipse and can have at most four points in common with the euclidian unit circle of $P$ except if it is equal to this circle, in which case $|x|=|y|=1$.

We now define the following formula $N_{\theta}(x)$ with free variable $x$

$$
\exists y\left(y \neq x \wedge_{0<k_{1}<\cdots<k_{5}<m}\left(\Lambda_{i=1}^{5} \delta\left(\cos \left(k_{i} \theta\right) x+\sin \left(k_{i} \theta\right) y\right)\right)\right)
$$

where $\theta$ is a real number such that $0<\theta<2 \pi / 5, \cos \theta, \sin \theta \in Q$ and $m$ is the least integer such that $m>2 \pi / \theta$.

Actually, the formula $N_{\theta}(x)$ is not a formula of $L$, but it is easy, using Lemma 2.5 to find a formula of $L$ equivalent to $N_{\theta}(x) . N_{\theta}(x)$ means that, for some $y$, at least five elements $(\cos k \theta) x+(\sin k \theta) y$ are extreme points of the unit cell; thus clearly, by Lemma 2.7, $X \vDash N_{\theta}(b)$ implies $|b|=1$.

We now let $\varphi_{\theta}(x)$ be the formula

$$
B((1-\delta) x) \wedge N_{\theta}(x) \text {. }
$$

LEMMA 2.9. Assume $X \vDash \varphi_{\theta}(b)$, then $b$ is equal to some element $\varepsilon e_{q^{*},} \varepsilon= \pm 1$, $q^{*} \in \mathbf{Q}^{*}$.

Proof. $X \vDash \varphi_{\theta}(b)$ implies

$\|b\|<(1-\delta)^{-1}$,

$|b|=1$.

Hence, by Lemma 2.3, $b=\varepsilon e_{q^{*}} \varepsilon= \pm 1, q^{*} \in Q^{*}$.

In order to prove a converse to Lemma 2.9 , it is necessary to fix $\theta$. Recall Lemma 2.4. Let $\psi, \psi^{\prime}$ be defined by

$$
0<\psi<\pi / 2 ; 0<\psi^{\prime}<\pi / 2, \quad \cos \psi=\alpha ; \cos \psi^{\prime}=\beta,
$$

where $\alpha, \beta$ are as in Lemma 2.4; clearly $\psi<\psi^{\prime}$.

We let $\theta$ be any fixed real number such that $0<\theta<\left(\psi^{\prime}-\psi\right) / 6$ and $\cos \theta, \sin \theta \in \mathbf{Q}$. This is possible in view of the following fact.

FACT. For any $\varepsilon>0$, there exists $\theta, 0<\theta<\varepsilon$, such that $\cos \theta, \sin \theta \in \mathbf{Q}$.

PROOF OF THE FACT. Let $i$ be an integer such that $2 i+1$ is a square (say $n^{2}$ )

$$
\left(\frac{i}{i+1}\right)^{2}+\frac{n^{2}}{(i+1)^{2}}=\frac{i^{2}+n^{2}}{(i+1)^{2}}=\frac{i^{2}+2 i+1}{(i+1)^{2}}=1 .
$$

so that there is an angle $\theta$ with $0<\theta<\pi / 2$ and

$$
\sin \theta=\frac{n}{i+1}, \quad \cos \theta=\frac{i}{i+1} .
$$

Now, when $i \rightarrow \infty, \cos \theta \rightarrow 1$ hence $\theta \rightarrow 0$.

From now on, we assume $\theta$ is fixed as indicated above.

Lema 2.10. Assume $e_{q^{*}}$ belongs to $X$ then $X \vDash \varphi_{\theta}\left(e_{q^{*}}\right)$ and $X \vDash \varphi_{\theta}\left(-e_{q^{*}}\right)$. 
Proof. Pick an element $y$ such that $|y|=1,\left(y, e_{q^{*}}\right)=0$, and $y \in X$ and consider the sequence $u_{k}=(\cos k \theta) e_{q^{*}}+(\sin k \theta) y, k<m$, where $m$ is the least integer such that $m \theta \geqslant 2 \pi$.

Because $0<\theta<\left(\psi-\psi^{\prime}\right) / 6$ at least five consecutive integers say $k, k+$ $1, \ldots, k+4$ are such that

$$
\psi<(k+i) \theta<\psi^{\prime} ; \quad i=0,1, \ldots, 4,
$$

it follows that

$$
\alpha>\cos (k+i) \theta>\beta ; \quad i=0,1, \ldots, 4,
$$

so that

$$
\alpha>\left(e_{q^{*}}, u_{k+i}\right)>\beta ; \quad i=0,1, \ldots, 4,
$$

hence by Lemma 2.4 ,

$$
\left\|u_{k+i}\right\|=1 ; \quad i=0,1, \ldots, 4 .
$$

By Lemma 2.7, $u_{k+i}, 0<i<4$, is an extreme point of the unit ball of $X$, so that $X \vDash N_{\theta}\left(\varepsilon e_{q^{*}}\right)$ but $\left\|e_{q^{*}}\right\|=(1-\delta)^{-1}$ i.e. $X \vDash B\left((1-\delta) e_{q^{*}}\right)$; finally, one gets $X \vDash \varphi_{\theta}\left(e_{q^{*}}^{\dagger}\right)$. Similarly $X \vDash \varphi_{\theta}\left(-e_{q^{*}}\right)$.

So far, we have studied relations which could be defined by a single formula $\varphi\left(x_{1}, \ldots, x_{n}\right)$ in every infinite dimensional closed subspace $X$ of any ultrapower $M^{I} /$ थ. From now on, we make an extra assumption on $X$, and we study relations which can be defined by a single formula in those spaces $X$ which satisfy this assumption. The extra assumption is the following:

$$
\forall n \quad X \vDash \exists x_{1} \cdots \exists x_{n}\left(\bigwedge_{i \neq j} x_{i} \neq x_{j} \wedge \bigwedge_{1<i<n} \varphi_{\theta}\left(x_{i}\right)\right) .
$$

It means that $X$ contains infinitely many elements $e_{q^{*}}, q^{*} \in Q^{*}$, and it will be shortened by $X \vDash \exists^{\infty} x \varphi_{\theta}(x)$. In order to emphasize the fact that definable relations are considered only for those spaces $X$ which satisfy $\exists^{\infty} x \varphi_{\theta}(x)$, the statements of the lemmas will begin by the words: Assume $X \vDash \exists^{\infty} x \varphi_{\theta}(x)$. For example:

LEMMa 2.11. Assume $X \vDash \exists^{\infty} x \varphi_{\theta}(x)$; then the relation $|b|=1$ is definable.

Proof. We claim $|b|=1$ if and only if $X \vDash N_{\theta}(b)$. Clearly, $X \vDash N_{\theta}(b)$ implies $|b|=1$. To show the converse implication, let $|b|=1$; pick $q^{*} \in \mathbf{Q}^{*}$ such that $X$ ₹ $\varphi_{\theta}\left(\varepsilon e_{q^{*}}\right), \varepsilon= \pm 1$. Now, if $b$ is $e_{q^{*}}$ or $-e_{q^{*}}$, then $X \vDash N_{\theta}(b)$; if not there is an element $c$ such that

$|c|=1$,

$(c, b)=0$,

$c$ belongs to the plane spanned by $e_{q^{*}}$ and $b$.

Then exactly as in Lemma 2.10, one can see that at least five of the points

$$
t_{k}=(\cos k \theta) b+(\sin k \theta) c ; \quad 0<k<m,
$$


are such that $\alpha>\left(t_{k}, e_{q^{*}}\right)>\beta$. Hence, by the same argument one concludes that $X \vDash N_{\theta}(b)$.

Corollary 2.12. Assume $X \vDash \exists^{\infty} x p_{\theta}(x)$; then the relation $|b|<1$ is definable.

Proof. $|b|<1$ if and only if there exist $c, c^{\prime}, c \neq c^{\prime}$ such that $|c|=1$ and $\left|c^{\prime}\right|=1$ and $\left(c+c^{\prime}\right) / 2=b$. Therefore $|b|<1$ is defined by

$$
\exists y \exists y^{\prime} \quad\left(N_{\theta}(y) \wedge N_{\theta}\left(y^{\prime}\right) \wedge y \neq y^{\prime} \wedge y+y^{\prime}=2 x\right) .
$$

Our next task is to get-in a definable way-a basis of neighborhoods of 0 in $X$.

In order to do so, we consider the set $C_{X}=\{b:|b|<1$ and $\|b\|>1\}$, and the relation $V\left(c, c^{\prime}\right)$, given by $c \in C_{X}$ and $c^{\prime} \in C_{X}$ and $2 c-c^{\prime} \in C_{X}$.

Obviously, by Corollary 2.12, we get

LEMMA 2.13. Assume $X \vDash \exists^{\infty} x \varphi_{\theta}(x)$; then the relation $V\left(c, c^{\prime}\right)$ is definable.

Moreover, we have:

Lemma 2.14. Assume $X \vDash \exists^{\infty} x \varphi_{\theta}(x)$; then, when $c$ varies over $C_{X},\left\{c^{\prime}-c\right.$ : $\left.V\left(c, c^{\prime}\right)\right\}$ is a basis for the neighborhoods of 0 .

Proof. First let $c$ be a given element of $C_{X}$; we have $|c|<1$ and $\|c\|>1$, so that for some $a \in A^{\prime}$ and some $\varepsilon= \pm 1,(c, \varepsilon a)>1-\Delta(a)$.

Let $\eta$ be such that $\left|c^{\prime}-c\right|<\eta$ implies $\left|c^{\prime}\right|<1$ and $\left(c^{\prime}, \varepsilon a\right)>1-\Delta(a)$; then $\left\|c^{\prime}-c\right\|<\eta$ implies $\left|c^{\prime}-\dot{c}\right|<\eta$ (by Lemma 2.3) and also $\mid\left(2 c-c^{\prime}\right)-$ $c|=| c-c^{\prime} \mid<\eta$ so that both $c^{\prime}$ and $2 c-c^{\prime}$ are in $C_{X}$. Thus

$$
\left\{c^{\prime}-c: V\left(c, c^{\prime}\right)\right\} \supseteq\left\{c^{\prime}-c:\left\|c^{\prime}-c\right\|<\eta\right\}
$$

so that it is a neighborhood of 0 .

Conversely, let $\eta>0$ be given; pick $a \in A^{\prime}$ such that $a \in X$ and $16 \Delta(a)<$ $\eta^{2}$. This is possible because $X$ contains infinitely many elements $e_{q^{*}}$, so that one can let $a$ be equal to $a_{\sigma}$ for any sequence $q_{1}^{*}>\cdots>q_{n}^{*}$ such that $e_{q^{*}} \in X, i=1, \ldots, n$, and $n$ is large enough.

Let $c$ be $a(1-\Delta(a) / 2)$ and assume $V\left(c^{\prime}, c\right)$ holds, then $\left|2 c-c^{\prime}\right|<1$ so that $\left(2 c-c^{\prime}, a\right)<1$ but $\left(2 c-c^{\prime}, a\right)=2(c, a)-\left(c^{\prime}, a\right)=2-\Delta(a)-\left(c^{\prime}, a\right)$ so that $\left(c^{\prime}, a\right)>1-\Delta(a)$. From this, we get

$$
\begin{aligned}
\left|c^{\prime}-a\right|^{2} & =\left|c^{\prime}\right|^{2}+|a|^{2}-2\left(a, c^{\prime}\right) \\
& <2-2\left(c^{\prime}, a\right)<2 \Delta(a)
\end{aligned}
$$

also

$$
|c-a|=\Delta(a) / 2<\Delta(a)<\sqrt{2 \Delta(a)}
$$

so that $\left|c-c^{\prime}\right|<\sqrt{2 \Delta(a)}+\sqrt{2 \Delta(a)}<4 \sqrt{\Delta(a)} \leqslant \eta$, this finishes the proof of Lemma 2.14. 
If $c$ is a given element of $C_{X}$, we let $V_{c}$ be $\left\{c^{\prime}-c: V\left(c, c^{\prime}\right)\right\}$; clearly if we assume $X \vDash \exists^{\infty} x \varphi_{\theta}(x)$, then the relation $b \in V_{c}$ is definable.

Lemma 2.15. Assume $X \vDash \exists^{\infty} x \varphi_{\theta}(x)$; then the relation $\|b\|<1$ is definable.

Proof. Clearly, it is enough to show that $\|b\|=1$ is definable. But in view of Lemma 2.14, $\|b\|=1$ iff $\|b\|<1$ and $\forall c \in C_{x} \exists y\left(y \in V_{c}\right.$ and $\|b+y\|$ $>1$ ) hence $\|b\|=1$ is definable.

LEMma 2.16. Assume $X \vDash \exists^{\infty} x \varphi_{\theta}(x)$; then the relation " $b$ is the center of $a$ facet of the unit ball of $X$ " is definable.

Proof. Assume $b$ belongs to a facet of the unit ball of $X$, say $X \cap$ $F(\varepsilon a, \Delta(a)), a \in A^{\prime}, \varepsilon= \pm 1$; let $E_{b}=\{d: d$ is an extreme point of the unit ball of $X$ and $\|b+d\| / 2=1$ \}. Clearly $E_{b}$ is the intersection of the unit ball of $X$ with the hyperplane $H(\varepsilon a, \Delta(a))$, so that if $b$ is the center of the facet $\forall x \in E_{b} 2 b-x \in E_{b}$. Conversely assume $b$ is such that

$\|b\| \neq 1$,

$|b|<1$,

$E_{b}$ is not empty (say $d \in E_{b}$ ),

$\forall x \in E_{b} 2 b-x \in E_{b}$,

then $d,(d+b) / 2, b$ are three distinct points in the unit sphere of $X$ which lie on the same line, so that $b$ belongs to some facet $F(\varepsilon a, \Delta(a)) \cap X$. Moreover, as $E_{b}$ is invariant under the symmetry $x \rightarrow 2 b-x$, then $b$ is the center of the facet $X \cap F(\varepsilon a, \Delta(a))$.

From these remarks the conclusion of the lemma follows.

COROLLARY 2.17. Assume $X \vDash \exists^{\infty} x \varphi_{\theta}(x)$; then the relation " $b$ is the limit of an infinite sequence of centers of facets of the unit ball of $X$ " is definable by $a$ formula $\nu(x)$.

Proof. Just write that for any $c$ in $C_{X}$ there exists an element $d$ of $V_{c}$ such that $d \neq 0$ and $b+d$ is the center of a facet.

Lemma 2.18. Assume $X \vDash \exists^{\infty} x \varphi_{\theta}(x)$, then the relation $|b|=|c|=1$ and $(b, c)=0$ is definable in $X$.

Proof. Assume $|b|=|c|=1$ then $\left|\frac{3}{5} b+\frac{4}{5} c\right|^{2}=1+\frac{24}{25}(b, c)$, so that $(b, c)=0$ iff $\left|\frac{3}{5} b+\frac{4}{5} c\right|=1$.

LEMMA 2.19. Assume $X \vDash \exists^{\infty} x \varphi_{\theta}(x)$, then the property " $X$ is the closed linear span of those $e_{q^{*}}$ it contains" is definable by a formula $\lambda$.

Proof. $\lambda$ is a formula of $L$ equivalent to $\forall z\left(|z|=1 \rightarrow \exists x\left(\varphi_{\theta}(x) \wedge(x, z)\right.\right.$ $\neq 0$ )) (such a formula exists by Lemma 2.18).

If $\lambda$ does not hold, $X$ contains an element which is orthogonal to all the $e_{q^{*}}$ included in $X$, therefore $X$ is not the closed linear span of those $e_{q^{*}}$. 
Conversely, if $X$ is not the closed linear span of those $e_{q^{*}}$ it contains, then, there is an element $c$ such that

$$
\begin{aligned}
& |c|=1, \\
& \left(c, e_{q^{*}}\right)=0 ; e_{q^{*}} \in X .
\end{aligned}
$$

Therefore $\lambda$ does not hold.

3. Proof of the main theorem. In order to define the set of sentences $\Phi$ of the main theorem, we first show how to describe by a set of formulas the subspaces of the ultrapowers of $M$.

Let $\hat{L}$ be a first order language including, besides variables,

a binary function symbol + ,

a unary function symbol $q$, for any $q \in \mathbf{Q}$,

a unary predicate symbol $B$,

a unary predicate symbol $B^{\prime}$.

Any real Banach space $E$ can be viewed as an $\hat{L}$-structure $\mathscr{A}(E) ;+$ is interpreted by the addition in $E, q$. by the multiplication by the scalar $q, B$ by the unit ball of $E$, and $B^{\prime}$ by the set of elements of $E$ of norm $\geqslant 1$.

By a term of $\hat{L}$, we mean as usual an expression like $q_{1} \cdot x_{1}+\cdots+q_{n} \cdot x_{n}$ where $x_{1}, \ldots, x_{n}$ are variables.

The following is implicit in [3] as well as [4].

Proposition 3.1. $X$ is a subspace of an ultrapower of $M$ if and only if $X$ satisfies all the formulas

$$
\forall x \cdots \forall x_{n} \quad\left(\bigvee_{i=1}^{k} B\left(t_{i}\right) \bigvee \bigvee_{j=1}^{l} B^{\prime}\left(u_{j}\right)\right)
$$

where $t_{1}, \ldots, t_{k} ; u_{1}, \ldots, u_{l}$ are terms with free variables among $x_{1}, \ldots, x_{n}$ and

$$
M \vDash \forall x_{1} \cdots \forall x_{n}\left(\bigvee_{i=1}^{k} B\left(t_{i}\right) \bigvee \bigvee_{j=1}^{l} B^{\prime}\left(u_{j}\right)\right)
$$

Before proving the proposition, we note the following.

COROLLARY 3.2. There is a set of formulas $\Phi_{0}$ of $L$ such that a given Banach space $X$ is a subspace of an ultrapower of $M$ if and only if $X \vDash \Phi_{0}$.

Proof of Corollary. By Proposition 3.1, $X$ is a subspace of an ultrapower of $M$, if and only if $X$ satisfies a set of formulas $\Psi$ of $\hat{L}$ of the form

$$
\forall x_{1} \cdots \forall x_{n} \quad\left(\bigvee_{i=1}^{k} B\left(t_{i}\right) \vee \bigvee_{j=1}^{\prime} B^{\prime}\left(u_{j}\right)\right)
$$

Now the models of $\Psi$ are exactly the models of $\Psi^{\prime}$ where $\Psi^{\prime}$ is obtained from $\Psi$ by replacing the formula 


$$
\forall x_{1} \cdots \forall x_{n} \quad\left(\bigvee_{i=1}^{k} B\left(t_{i}\right) \vee \bigvee_{j=1}^{\prime} B^{\prime}\left(u_{j}\right)\right)
$$

by the infinite set

$$
\left.\forall x_{1} \cdots \forall x_{n}\left(\bigvee_{i=1}^{k} B\left(t_{i}\right) \vee \bigvee_{j=1}^{l}\right\urcorner B\left(q u_{j}\right)\right), \quad q>1 .
$$

Using the technique of Lemma 2.5 and Corollary 2.6, it is easy to translate $\Psi^{\prime}$ into a set of formulas $\Phi_{0}$ of $L$.

We now have to prove Proposition 3.1. In order to do so, we recall the following observation from $[4, \S 5]$.

OBSERVATION. $E^{I} / \mathfrak{U}$ is obtained from the usual ultrapower $\mathfrak{B}=\mathfrak{U}(E)^{I} / \mathfrak{U}$ by performing the following operations:

First one restricts $\mathfrak{B}$ to those elements $b$ such that for some $q \neq 0$, $\mathfrak{B} \vDash B(q \cdot b)$, thus one gets a substructure $\mathfrak{B}_{f}$.

Then, one takes the quotient of $\mathfrak{B}_{f}$ by the equivalence relation $b \sim b^{\prime}$ defined by: for any $q \in \mathbf{Q}, \mathfrak{B} \vDash B\left(q\left(b-b^{\prime}\right)\right)$.

Finally, one defines the norm by $\|b\|=(\sup \{q: \mathfrak{B} \vDash B(q b)\})^{-1}$.

From this observation, it follows that the truth of any positive universal formula of $\hat{L}$ is preserved if one goes from $M$ to a subspace of $M^{I} / \mathcal{U}$.

Conversely, to embed isometrically a Banach space $X$ into an ultrapower $M^{I} / \mathcal{U}$, it is enough to find a mapping $\theta: X \rightarrow \mathfrak{U}(M)^{I} / \mathcal{U}$ such that for any sequence $a_{1}, \ldots, a_{n}$ of elements of $X$, any sequence of rational numbers $q_{1}, \ldots, q_{n}$ and any rational number $\varepsilon, 1>\varepsilon>0$,

$$
\left\|a_{1} q_{1}+\cdots+a_{n} q_{n}\right\|<1 \text { implies } 7 B^{\prime}\left(\frac{\theta\left(a_{1}\right) q_{1}+\cdots+\theta\left(a_{n}\right) q_{n}}{1+\varepsilon}\right)
$$

and

$$
\left\|a_{1} q_{1}+\cdots+a_{n} q_{n}\right\|>1 \text { implies } 7 B\left(\frac{\theta\left(a_{1}\right) q_{1}+\cdots+\theta\left(a_{n}\right) q_{n}}{1-\varepsilon}\right) .
$$

If this is not possible, then in the language $\hat{L}[X]$ obtained by adding a constant symbol a for any $a$ in $X$, the following set of formulas is not consistent:

$$
\begin{aligned}
& \operatorname{Th}(\mathfrak{A}(M)) \quad \text { (the set of all closed formulas true in } \mathfrak{A}(M)) . \\
& 7 B^{\prime}\left(\frac{a_{1} q_{1}+\cdots+a_{n} q_{n}}{1+\varepsilon}\right) ; \quad\left\|a_{1} q_{1}+\cdots+a_{n} q_{n}\right\|<1 . \\
& 7 B\left(\frac{a_{1} q_{1}+\cdots+a_{n} q_{n}}{1-\varepsilon}\right) ; \quad\left\|a_{1} q_{1}+\cdots+a_{n} q_{n}\right\|>1 .
\end{aligned}
$$

So that there exists a finite sequence of terms $t_{1}, \ldots, t_{k}, u_{1}, \ldots, u_{l}$ depending on $a_{1}, \ldots, a_{n}$ such that 


$$
\begin{gathered}
\operatorname{Th}(\mathfrak{U}(M))+\bigvee_{i=1}^{k} B\left(\frac{t_{i}}{1-\varepsilon_{i}}\right) \vee \bigvee_{j=1}^{l} B^{\prime}\left(\frac{u_{j}}{1+\varepsilon_{j}^{\prime}}\right), \\
t_{i}\left(a_{1}, \ldots, a_{n}\right) \geqslant 1, \quad 1<i<k, \\
u_{j}\left(a_{1}, \ldots, a_{n}\right)<1, \quad 1<j<l .
\end{gathered}
$$

Substituting $x_{1}, \ldots, x_{n}$ for $a_{1}, \ldots, a_{n}$, we get

$M \vDash \forall x_{1} \cdots \forall x_{n}\left(\bigvee_{i=1}^{k} B\left(\frac{t_{i}\left(x_{1}, \ldots, x_{n}\right)}{1-\varepsilon_{i}}\right) \vee \bigvee_{j=1}^{\prime} B^{\prime}\left(\frac{u_{j}\left(x_{1}, \ldots, x_{n}\right)}{1+\varepsilon_{j}^{\prime}}\right)\right)$.

Hence, if the hypothesis of Proposition 3.1 holds, we get

$$
X \vDash \forall x_{1} \cdots \forall x_{n}\left(\bigvee_{i=1}^{k} B\left(\frac{t_{i}}{1-\varepsilon_{i}}\right) \vee \bigvee_{j=1}^{\prime} B^{\prime}\left(\frac{u_{j}}{1+\varepsilon_{j}^{\prime}}\right)\right)
$$

but $a_{1}, \ldots, a_{n}$ contradict this last formula; this finishes the proof of the proposition.

We now define $\Phi, \varphi(x), \psi(x, y), \theta . \Phi$ includes

the formulas of $\Phi_{0}$ (defined in Corollary 3.2);

the formulas

$$
\exists x_{1} \cdots \exists x_{n} \quad \bigwedge_{i} B\left(x_{i}\right) \wedge \bigwedge_{i \neq j} 7 B\left(x_{i}-x_{j}\right), \quad n \in \mathbf{N}
$$

(these formulas hold in $X \subseteq M^{I} / \mathcal{Q}$ if and only if $X$ is infinite dimensional); the formulas

$$
\exists x_{1} \cdots \exists x_{n}\left(\bigwedge_{i=1}^{n} \varphi_{\theta}\left(x_{n}\right) \wedge \bigwedge_{i \neq j} x_{i} \neq x_{j}\right)
$$

(these formulas mean that infinitely many $e_{q^{*}} ; q^{*} \in \mathrm{Q}^{*}$, are included in $X$ ); the formula $\lambda$ given by Lemma 2.19 (and meaning that $X$ is the closed linear span of those $e_{q^{*}}$ it includes).

$\varphi(x)$ is the formula $\varphi_{\theta}(x)$,

$\psi(x, y)$ is $\varphi_{\theta}(x) \wedge \varphi_{\theta}(y) \wedge(x=y \vee x=-y \vee\|x+2 y\|>r)$

where $r$ is a fixed rational number with $\sqrt{5}<r<\sqrt{5}\left(1-\delta_{1}\right)^{-1}$;

$\psi(x, y)$ means that $x=\varepsilon e_{q^{*}}, \varepsilon= \pm 1, q^{*} \in Q^{*}, y=\varepsilon^{\prime} e_{p^{*}}, \varepsilon^{\prime}= \pm 1, p^{*} \in$ $Q^{*}$ and $q^{*}<p^{*}$.

$\theta$ is the formula $7 \exists x \nu(x)$, where $\nu(x)$ is defined in the proof of Corollary 2.17 and is such that $X \vDash \nu(b)$ iff $b$ is the limit of an infinite sequence of centers of facets of the unit ball of $X$ (this will be henceforth abbreviated by " $b$ is a limit point of facets").

Let $E$ be a model of $\Phi$; then, by Corollary 3.2, there exist $I$, $U$ such that $E$ is isometric to a subspace of $M^{I} / \mathcal{U}$. Without loss of generality we may assume $E \subseteq M^{I} / थ$. Clearly, $E$ is spanned by those $e_{q^{*}}$ it includes (because $E \vDash \lambda)$ and $D(E)=\{a \in E: \varphi(a)\}$ is infinite. 
Now the quotient of $D(E)$ by the equivalence relation $a= \pm b$ is ordered by $\psi(x, y)$. We let $C(E)$ denote this ordering.

Actually $C(E)$ is a linear ordering: given any pair of elements $a, a^{\prime}$ such that $E$ F $\varphi(a), E \vDash \varphi\left(a^{\prime}\right)$ one can find $\varepsilon= \pm 1, \varepsilon^{\prime}= \pm 1, q^{*} \in \mathbf{Q}^{*}, p^{*} \in \mathbf{Q}^{*}$ with $a=\varepsilon e_{q^{*}}, a^{\prime}=\varepsilon^{\prime} e_{p^{*}}$ then either $p^{*}=q^{*}$ or $p^{*}<q^{*}$ or $q^{*}<p^{*}$. So that, either $E \vDash \psi\left(a, a^{\prime}\right)$ or $E \vDash \psi\left(a^{\prime}, a\right)$.

Conversely let $(C, \leqslant)$ be an infinite linear ordering; it is known that for some ultrafilter $\mathcal{U}$ on a set $I(C,<)$ can be embedded into $Q^{I} / \mathcal{U}=Q^{*}$. Let $i: C \rightarrow Q^{*}$ be such an embedding. It is easy to see that the subspace $E$ of $M^{I} / \mathcal{Q}$ spanned by the set $\left\{e_{i(c)}: c \in C\right\}$ is a model of $\Phi$ and that $C(E)$ is precisely isomorphic to $(C, \varangle)$.

To complete the proof of the main theorem, it is enough to show the following:

LEMMA 3.3. $C(E)$ is a well ordering if and only if no element of the unit ball of $E$ is a limit point of facets.

Proof. Assume first $C(E)$ is not a well ordering. Let $\left(a_{n}\right)_{n \in N}$ be an infinite decreasing sequence, let

$$
a_{n}=\varepsilon_{n} e_{q_{n}^{*}}, \quad \varepsilon_{n}= \pm 1, \quad q_{n}^{*} \in \mathrm{Q}^{*}
$$

(as usual $E$ is considered as a subspace of $M^{I} / थ$ ).

Then if $\sigma_{n}^{*}=\left\{q_{1}^{*}, \ldots, q_{n}^{*}\right\}, n \geqslant 3$, the sequence $\left(\left(1-\delta_{n}\right) a_{\sigma_{n}^{*}}\right)_{n \in \mathrm{N}}$ is an infinite sequence of centers of facets with limit

$$
\left(\sum_{i=1}^{\infty} e_{q_{i}^{*}} / i\right)\left(\sum_{j=1}^{\infty} \frac{1}{j^{2}}\right)^{-1 / 2} \text {. }
$$

Conversely, let $x$ be the limit of an infinite sequence of centers of facets say $\left(c_{m}\right)_{m \in N^{*}}$ Obviously

$$
\lim _{\substack{m \rightarrow \infty \\ m \rightarrow \infty}}\left|c_{m}-c_{m^{\prime}}\right| \rightarrow 0
$$

so that by Lemma 2.2, for $m$ large enough, $c_{m}$ is of the form $\varepsilon_{m} a_{\sigma_{m}^{*}}\left(1-\delta_{l\left(\sigma_{m}^{*}\right)}\right)$, $\sigma_{m}^{*} \in Q^{*<\omega}, \varepsilon_{m}= \pm 1$, with $\lim l\left(\sigma_{m}^{*}\right)=\infty$.

Now if $\sigma^{*}=\left\{q_{1}^{*}, \ldots, q_{k}^{*}\right\}, q_{1}^{*}>\cdots>q_{k}^{*}$, and $\tau^{*}=\left\{q_{1}^{\prime *}, \ldots, q_{j}^{* *}\right\}$, $q_{1}^{*}>\cdots>q_{j}^{\prime *}$, we define as in Lemma $2.3, n\left(\sigma^{*}, t^{*}\right)$ to be the least integer such that $q_{i}^{*} \neq q_{i}^{\prime *}$ or $i>k$ or $i>j$.

By Lemma 2.2, as

one gets $n\left(\sigma_{m}^{*}, \sigma_{m}^{*}\right) \rightarrow \infty$.

$$
\left|\varepsilon_{m} a_{\sigma_{m}^{*}}-\varepsilon_{m^{\prime}} a_{\sigma_{m^{\prime}}}\right| \rightarrow 0
$$

This means exactly that the finite decreasing sequence $\left(\sigma_{m}^{*}\right)_{m \in Q}$ has a pointwise limit which is an infinite decreasing sequence. Furthermore, given any $m$ and any element $q^{*}$ of the sequence $\sigma_{m}^{*}$, we have $\left(a_{\sigma_{m}^{*}}, e_{q^{*}}\right) \neq 0$, hence, 
as $E$ is the closed linear span of these $e_{q^{*}}$ it contains, $e_{q^{*}} \in E$; finally, this shows that $C(E)$ is not a well ordering.

4. The notion of completeness. In this section, we show that "completeness" is a notion which bears all the strength of second order logic with second order variables ranging over (at most) countable sets.

We first indicate briefly how to adapt $\$ \$ 1,2,3$ in order to get a proof of the general theorem (stated on page ). The basic model $M$ must be replaced by a slightly different one $M^{\prime}$ which we now describe. $M^{\prime}$ is a space $\mathcal{S C}(A, \Delta, \delta)$ for suitable $\Delta, A, \delta$.

$\mathcal{X}$ is the space $l^{2}\left(\mathrm{~N} \cup \mathrm{N}^{2}\right)$. We let $m, n$ range over integers. $e_{n}$ is the element of $\mathcal{X}$ whose value is 1 at $n, 0$ elsewhere. Similarly for $e_{n, m}$. For any one-one sequence $\sigma=\left\{n_{1}, \ldots, n_{k}\right\}$, we let

$$
a_{\sigma}=\left(e_{n_{1}}+\frac{e_{n_{2}}}{2}+\cdots+\frac{e_{n_{k}}}{k}\right)\left(\sum_{i=1}^{k} \frac{1}{i^{2}}\right)^{-1 / 2}
$$

$k=l(\sigma)$ is the length of $\sigma$.

$A$ consists of the following points of $\mathrm{IC}$ :

$e_{n}, n \in \mathrm{N}$.

$e_{n, m}, n \in \mathbf{N}, m \in \mathbf{N}$.

$\left(e_{n}+2 \varepsilon e_{n, m}\right) / \sqrt{5}, n \in \mathrm{N}, m \in \mathrm{N}, \varepsilon= \pm 1$.

$\left(2 e_{m}+\varepsilon e_{n, m}\right) / \sqrt{5}, n \in \mathrm{N}, m \in \mathrm{N}, \varepsilon= \pm 1$.

$a_{\sigma}, \sigma=\left\{n_{1}, \ldots, n_{k}\right\}, l(\sigma)>3$.

$\Delta$ is defined by

$\Delta\left(e_{n}\right)=\delta_{0}, n \in \mathrm{N}$.

$\Delta\left(e_{n, m}\right)=\delta_{1}, n \in \mathrm{N}, m \in \mathbf{N}$.

$\Delta\left(\left(e_{n}+2 \varepsilon e_{n, m}\right) / \sqrt{5}\right)=\delta_{2}, n \in \mathrm{N}, m \in \mathrm{N}, \varepsilon= \pm 1$.

$\Delta\left(\left(2 e_{m}+\varepsilon e_{n, m}\right) / \sqrt{5}\right)=\delta_{2}, n \in \mathbf{N}, m \in \mathbf{N}, \varepsilon= \pm 1$.

$\Delta\left(a_{o}\right)=\delta_{l(o)}$

where $\delta_{n}$ is an infinite decreasing sequence like in \$1. (Actually, for technical reasons, the sequence $\delta_{n}$ should decrease more rapidly than in $\$ 1$; we skip the details.)

.The idea of the proof is the following: given a subspace $X$ of an ultrapower $M^{\prime I} / \mathcal{Q}$ we want to define a structure $C(X)$ whose domain is the quotient of $\left\{\varepsilon e_{n^{*}} \in X: n^{*} \in N^{I} / \mathcal{Q}, \varepsilon= \pm 1\right\}$ by the relation $a= \pm b$, and whose binary relation $R_{X}$ is given by

$$
\left(e_{n^{*}}, e_{m^{*}}\right) \in R_{X} \text { iff } e_{n^{*}, m^{*}} \in X .
$$

For this reason we define a set of formulas $\Phi$ of $L$ in such a way that the Banach spaces $X$ which model $\Phi$ have the following properties:

(1) $X$ is a subspace of some ultrapower of $M^{\prime}$, say $M^{\prime \prime} /$.

(2) $X$ is infinite dimensional. 
(3) $X$ contains infinitely many $e_{n^{*}}, n^{*} \in \mathrm{N}^{I} /$ U.

(4) $X$ is the closed linear span of those $e_{n^{*}}$ it includes and of those $e_{n^{*}, m^{*}}$ such that $e_{m^{*}}, e_{n^{*}}, e_{n^{*}, m^{*}}$ belongs to $X$.

It is easy to express (1), (2), (3) using the same techniques as in $\$ \S 2$ and 3. To express (4), it is necessary to show that the set of elements $\varepsilon e_{n^{*}, m^{*}}$ included in $X$ as well as $e_{n^{*}}, e_{m^{*}}$, is definable. Actually this can be proved still using the same techniques, if one realizes that this set is precisely the set of elements $x$ of $X$ such that $|x|=1,\left(1-\delta_{1}\right) x$ is the center of a facet of the unit ball of $X$, there exist $e_{n^{*}}, e_{m^{*}}$ in $X$ such that

$$
\left\|e_{n^{*}}+2 x\right\|=\sqrt{5}\left(1-\delta_{2}\right)^{-1} \text { and }\left\|2 e_{m^{*}}+x\right\|=\sqrt{5}\left(1-\delta_{2}\right)^{-1} \text {. }
$$

In order to define the mapping $\Theta$ from $L^{\prime}(\omega)$ to $L$ which enables to translate the second order properties of the structure $C(X)$ into first order properties of the Banach $X$, a lemma is needed. To state this lemma, we let

$$
D(X)=\left\{\varepsilon e_{n^{*}}: \varepsilon= \pm 1, n^{*} \in \mathrm{N}^{I} / \mathcal{U}, e_{n^{*}} \in X\right\}
$$

LEMMA 4.1. There exists a formula of $L, \Omega(x, y)$ with two free variables such that

(i) Given any element $a$ of $X,\{b: X \vDash \Omega(a, b)\}$ is a symmetric subset of $D(X)$ which is at most countable.

(ii) Given any symmetric subset of $D(X)$, say $u$, which is finite or countable, there is an element $a$ of $X$ such that $u=\{b: X \vDash \Omega(a, b)\}$.

We will not give a detailed proof of this lemma. Essentially $\Omega(x, y)$ has the following meaning:

$y \in D(X)$ and the inner product $(x, y)$ is nonzero and one of the following happens

(1) either there exist $x_{1}, x_{2}$ in $D(X)$ such that $x=x_{1}+x_{2}$;

(2) or $x$ is the center of a facet of the unit ball of $X$;

(3) or else $x$ is a limit point of facets.

One can show that the points which are limit of an infinite sequence of facets are exactly

$$
\left\{\varepsilon a_{f}: \varepsilon= \pm 1, f \text { a one-one sequence of } \mathbf{N}^{I} / Q\right\}
$$

where $a_{f}$ stands for

$$
\left(\sum_{j=1}^{\infty} \frac{a_{f(j)}}{j}\right)\left(\sum_{i=1}^{\infty} \frac{1}{i^{2}}\right)^{-1 / 2} ;
$$

from this fact, it is clear that condition (3) above enables us to encode the countable symmetric subsets of $D_{X}$; conditions (1) and (2) take care of the finite subsets:

Using Lemma 4.1, the reader will easily supply a definition of the mapping 
$\Theta$, using induction on the length of formulas. (For example to start the induction $\Theta(R(x, y))$ can be any formula meaning: " $x$ is some $\varepsilon e_{n^{*}}, y$ is some $\varepsilon e_{m^{*}}$ and there exists $z$ which is some $\varepsilon e_{k^{*} j^{*}}$ and is such that

$$
\|x+2 z\|>r \text { and }\|2 y+z\|>r
$$

where $r$ is a rational number satisfying the inequalities $\sqrt{5}<r<\sqrt{5}(1-$ $\left.\delta_{2}\right)^{-1}$.)

To complete the circle of ideas discussed in the introduction, we now show that second order logic with second order variables ranging over at most countable sets actually expresses completeness.

THEOREM 4.2. There exists a formula $\gamma_{0}$ of $L(\omega)$ such that a normed space is a model of $\gamma_{0}$ if and only if it is a Banach space.

The proof requires a few tricks and therefore a few lemmas. In what follows, we let $x, y, z$ denote first order variables, $X, Y, Z$ second order variables.

LEMMA 4.3. There exists a formula $\theta(x, y)$ of $L(\omega)$ such that given any normed space $E$ together with elements $a, b, \theta(a, b)$ holds if and only if there exists $a$ positive real $\alpha$ such that $b=\alpha a$.

Proof. We first define a formula $\lambda(x, X), \lambda(x, X)$ is the conjunction of the following statements

(i) $X(x)$,

(ii) $\forall y(X(y+y) \rightarrow X(y))$,

(iii) $\forall y \forall z(X(y) \wedge X(z) \rightarrow X(y+z))$,

(iv) $X$ is the smallest countable set satisfying (i), (ii), (iii).

Clearly, given an element $a$ of $E$ and a countable subset $A, \lambda(a, A)$ holds iff $A=\left\{k a / 2^{m}: k \in \mathbf{N}, m \in \mathbf{N}\right\}$.

Now, $\theta(x, y)$ is given by

$\forall t \forall Y(\lambda(y, Y) \wedge Y(t)) \rightarrow \exists u \exists X(\lambda(x, X) \wedge X(u) \wedge B(t-u))$.

$\left(\theta(x, y)\right.$ means that the distance between $\left\{k x / 2^{m}\right\}$ and any element of $\left\{k y / 2^{m}\right\}$ is always bounded by 1 .)

LEMMA 4.4. There exists a formula $\kappa(x, y)$ of $L(\omega)$ whose meaning is $\|x\|<\|y\|$.

Proof. Let $\kappa_{0}(x, y)$ be the formula

\urcorner$\theta(y, x) \wedge\urcorner \theta(-y, x)$

$$
\left.\wedge \exists x^{\prime} \exists y^{\prime}\left(B\left(x^{\prime}\right) \wedge\right\urcorner B\left(y^{\prime}\right) \wedge \theta\left(x, x^{\prime}\right) \wedge \theta\left(y, y^{\prime}\right) \wedge \theta\left(x-y, x^{\prime}-y^{\prime}\right)\right) .
$$

Assume first $a, b$ linearly independent in $E$ with $\|a\|<\|b\|$. Then, if we let $\alpha$ be a positive real such that $\alpha\|a\|<1<\alpha\|b\|$, and $a^{\prime}=\alpha a, b^{\prime}=\alpha b$, it is easy 
to see that we have $7 \theta(a, b), 7 \theta(-b, a), B\left(a^{\prime}\right), 7 B\left(b^{\prime}\right), \theta\left(a, a^{\prime}\right), \theta\left(b, b^{\prime}\right)$, $\theta\left(a-b, a^{\prime}-b^{\prime}\right)$. So that $\kappa_{0}(a, b)$ holds.

Conversely, assume $\kappa_{0}(a, b)$ holds, then there exist $a^{\prime}, b^{\prime}$ and $\alpha, \beta, \gamma>0$ such that $a^{\prime}=\alpha a,\left\|a^{\prime}\right\|<1 ; b^{\prime}=\beta b,\left\|b^{\prime}\right\|>1 ; a^{\prime}-b^{\prime}=\gamma(a-b)$; but this gives $\alpha a-\beta b=\gamma(a-b)$ or $a(\alpha-\gamma)=b(\beta-\gamma)$. If $\alpha \neq \gamma$ then $a=$ $(\beta-\gamma) b /(\alpha-\gamma)$ so that either $\theta(b, a)$ or $\theta(-b, a)$ holds; contradiction. Hence $\alpha=\gamma$, this gives $b(\beta-\gamma)=0$ so that if $\beta \neq \gamma$ we get $b=0$ and also $b^{\prime}=\beta b=0$ but this contradicts $\left\|b^{\prime}\right\|>1$. Finally, $\alpha=\beta=\gamma$ and as $\|\alpha a\|<1<\|\alpha b\|$ we get $\alpha \neq 0$ and $\|a\|<\|b\|$.

To obtain $\kappa(x, y)$ we just consider the disjunction of the formulas $\kappa_{0}(x, y)$, $y \neq 0 \wedge \theta(y, x) \wedge \theta(y, y-x) \wedge x \neq y, y \neq 0 \wedge \theta(-y, x) \wedge \theta(-y,-y$ $-x) \wedge x \neq-y$.

LEMMA 4.5. There exists a formula $\delta(X)$ of $L(\omega)$ meaning $X$ is the range of an infinite norm increasing sequence.

Proor. $\delta(X)$ is a formula which says that the relation $\|x\|<\|y\|$ is a well ordering of $X$ such that any element has an immediate successor and any element different from the minimal one has an immediate predecessor. In view of the fact that $\|x\|<\|y\|$ can be expressed by $\kappa(x, y)$, such a formula $\delta(X)$ exists in $L(\omega)$.

We are now ready to prove Theorem 4.2. In order to find the formula $\gamma_{0}$ whose existence is given by the theorem, we consider the following property of a given space $E$ :

There exists a countable subset $X \subseteq E$ such that $X$ is the range of an infinite norm increasing sequence which is a Cauchy sequence and has no limit.

First of all, we claim that a normed space which is not a Banach space has this property. Indeed let $\left(x_{n}\right)_{n \in N}$ be a Cauchy sequence without a limit. Without loss of generality, we may assume

$$
\lim _{n \rightarrow \infty}\left\|x_{n}\right\| \neq 0 \text {. }
$$

Let

$$
y_{n}=\frac{x_{n}}{\left\|x_{n}\right\|}\left(1-\frac{1}{2^{n}}\right)
$$

Now, if $\alpha=\lim _{n \rightarrow \infty}\left\|x_{n}\right\|$, it is clear that

$$
\lim _{n \rightarrow \infty}\left[y_{n}-\frac{x_{n}}{\alpha}\right]=0
$$

so that $y_{n}$ is a Cauchy sequence without a limit. Clearly, $y_{n}$ is norm increasing. Hence it remains to translate the above property into a formula of $L(\omega)$. By Lemma 4.4, we already know a formula $\delta(X)$ meaning that $X$ is the range of 
an infinite norm increasing sequence. For such a sequence, the property of being a Cauchy sequence can be expressed by

$$
\begin{aligned}
\forall z \neq 0, \quad \exists x \quad X(x) \wedge \forall y \forall y^{\prime} \\
\left(X(y) \wedge X\left(y^{\prime}\right) \wedge \kappa(x, y) \wedge \kappa\left(x, y^{\prime}\right) \rightarrow \kappa\left(y-y^{\prime}, z\right)\right) .
\end{aligned}
$$

Finally, if $X$ is the range of an infinite norm increasing sequence, this sequence has a limit if and only if the following holds

$$
\exists t \quad \forall z \neq 0, \quad \exists x \quad X(x) \wedge \forall y \quad(X(y) \wedge \kappa(x, y) \rightarrow \kappa(t-x, z)) .
$$

Finally, we have shown that we could translate the property of not being complete by a formula $7 \gamma_{0}$ of $L(\omega)$.

This finishes the proof.

\section{REFERENCES}

1. D. Dacunha-Castelle and J. L. Krivine, Application des ultraproduits à l'étude des espaces et des algèbres de Banach, Studia Math. 41 (1972), 315-344.

2. J. L. Krivine, Langages à valuers reelles et applications, Fund. Math. 81 (1974), 213-253.

3. J. Stern, Some applications of model theory in Banach space theory, Ann. Math. Logic 9 (1976), 49-121.

4. Ultrapowers and local properties of Banach spaces, Trans. Amer. Math. Soc. 240 (1978), 231-252.

5. The problem of envelopes for Banach spaces, Israel J. Math. 24 (1970), 1-15.

6. C. Ward Henson, When two Banach spaces have isometrically isomorphic nonstandard hulls, Israel J. Math. (to appear).

7. M. Dickman, Large infinitary languages, North-Holland, Amsterdam, 1975.

8. J. Silver, Some applications of model theory in set theory, Ann. Math. Logic 3 (1971), 45-110.

Department of Mathematics, The Hebrew Untversity, Jerusalem, Israbi

U. E. R. de Mathématioue, Universití Paris Vil, Paris, France 